\title{
Clinical and epidemiological profile of Indian COVID-19 patients from Jaipur: a descriptive study
}

\author{
Sunil Mahavar, Princy Tyagi, Abhishek Agrawal, Sudhir Bhandari, Subrata Banerjee, Raman Sharma, \\ Prakash Keswani
}

Department of Medicine, Sawai ManSingh Medical College \& Hospital, Jaipur, India

\begin{abstract}
We analyzed the data of 102 confirmed patients with novel Coronavirus 2 infection (COVID-19) during the early period of nationwide lockdown announced in India after the declaration of pandemic. We analyzed epidemiological, clinical characteristics and outcome of hospitalization in 102 patients with positive results
\end{abstract}

Correspondence: Dr. Abhishek Agrawal, Senior Professor, Department of Medicine, Sawai ManSingh Medical College \& Hospital, Sawai Ramsingh Road, Jaipur 302 004, India.

Tel. +91.9829298691

E-mail: drabhie@yahoo.com

Keywords: COVID-19; severe acute respiratory syndrome coronavirus-2 (SARS-CoV-2); asymptomatic; clinical characteristic; India.

Contributions: All the authors of this manuscript have equally contributed to the concept, design, data collection, analysis and interpretation of the study as well as to the preparation of this manuscript. All the authors have approved the final version of the submitted manuscript and declare that they are equally responsible for all aspects of the submitted work.

Conflict of interest: The authors declare that they have no competing interests, and all authors confirm accuracy.

Funding: No funding has been received for this study from any source.

Ethics approval: Informed consent for data collection was obtained from each registered patient or from his guardian. During this pandemic, the Institutional COVID-19 Treatment Protocol Team has instructed the Institute to collect and analyze COVID-19 patients' data to determine various epidemiologic, clinical and laboratory features and possible factors associated with mortality; consequently, Institutional Ethics Committee approval was not needed. Furthermore, the data used is anonimysed and individual patient's identity is not revealed anywhere.

Received for publication: 13 May 2020.

Accepted for publication: 14 December 2020.

${ }^{\circ}$ Copyright: the Author(s), 2021

Licensee PAGEPress, Italy

Monaldi Archives for Chest Disease 2021; 91:1377

doi: 10.4081/monaldi.2021.1377

This article is distributed under the terms of the Creative Commons Attribution Noncommercial License (by-nc 4.0) which permits any noncommercial use, distribution, and reproduction in any medium, provided the original author(s) and source are credited. for novel corona virus (SARS-CoV-2) RNA testing which were traced on the basis of history of travel, contact with a confirmed COVID-19 case, resident of hotspot areas or presence of symptoms, thus providing an accurate estimate of the proportion of asymptomatic cases in the initial population. Of 102 patients enrolled in the study, $83.3 \%(85 / 102)$ were asymptomatic and $16.67 \%(17 / 102)$ were symptomatic. Seventy-seven $(75.49 \%)$ were males and $24.50 \%(25 / 102)$ were females. Eighteen $(17.6 \%)$ patients had associated comorbidities, the most prevalent of which were diabetes mellitus $10.8 \%$ (11/102), hypertension $7.8 \%(8 / 102)$, chronic obstructive pulmonary disease (COPD) in 3.92\% (4/102), chronic kidney Disease (CKD) $0.98 \%(1 / 102)$, coronary artery Disease (CAD) 0.98\% (1/102) and cerebro-vascular disease (CVD) $0.98 \%$ (1/102). The clinical spectrum among symptomatic COVID19 patients varied from dry cough and fever to respiratory failure and multi-organ failure. Twelve $(11.76 \%)$ patients were kept in intensive care unit (ICU). Ninety-nine (97.05\%) patients recovered while three $(2.94 \%)$ died during hospital stay. With majority of COVID-19 cases in India being asymptomatic, changes in biochemical and inflammatory profile were small and insignificant in asymptomatic patients when compared to symptomatic patients. Elevated NLR, lymphopenia, age and presence of comorbidities were associated with increased severity and poor outcome.

\section{Introduction}

In December 2019, an outbreak of unusual respiratory illness was recognized in Wuhan city, the capital of Hubei province in China, which was linked to a novel Coronavirus infection, initially named 2019-nCoV by World Health Organization (WHO) [1,2]. On February 11, 2020, WHO renamed the disease as coronavirus disease 2019 (COVID-19) [3]. On the basis of a phylogenetic analysis of related coronaviruses, 2019-nCoV was designated as severe acute respiratory syndrome coronavirus-2 (SARS-CoV-2) by the Coronavirus Study Group (CSG) of the International Committee on Virus Taxonomy [4]. On $11^{\text {th }}$ March 2020, COVID19 was declared to be a global pandemic by WHO and till date $15,538,736$ cases have been recorded and 634,325 have died so far worldwide [5]. The infection still continues to involve more communities and nations. Rajasthan, India detected its first case on $3^{\text {rd }}$ February 2020 in an Italian tourist and since then has recorded 34,178 cases till date of which 24,547 cases have recovered while 602 have died [6].

COVID-19 affects people of all age groups but elderly population (particularly $>60$ years) and those with underlying medical illness like cardiovascular disease, chronic respiratory disease or cancer are at higher risk of getting severe infection and 
poor outcome [7]. SARS-CoV-2 is highly contagious with rapid transmission even during the incubation period [8]. The clinical spectrum of SARS-CoV-2 infection appears to be wide encompassing asymptomatic infection, mild upper respiratory tract illness and severe viral pneumonia with respiratory failure and even death [9]. No approved drug regimen has been introduced to treat infected cases so far. A few antiviral drugs having different mechanisms of action have been used to alleviate the disease symptoms of these most promising is Remdesivir, which revealed its in vitro activity against the COVID19 virus and its safety was proven in Ebola trials [10]. Both chloroquine and hydroxychloroquine are believed to act on the entry and post-entry stages of SARS-CoV and SARS-CoV-2 infection, likely via effects on endosomal $\mathrm{pH}$ and the resulting underglycosylation of angiotensin converting enzyme 2 (ACE2) receptors that are required for viral entry [11]. Control strategies for outbreaks of Middle-East respiratory syndrome Coronavirus (MERS-CoV) infection have focused on tracing contacts of symptomatic patients with epidemiologic links. Knowledge of COVID-19 is still evolving, but anecdotal evidence is available suggesting that patients can be asymptomatic and remain infective for up to 14 days [12].

The aim of this study is to provide an insight into the initial COVID-19 cases that occurred as an outbreak in multiple clusters in the city of Jaipur, India by investigating patients with history of travel to endemic countries, resident of hotspot areas, recent respiratory symptoms and/or fever or who have been in contact with patients who have been previously tested positive for SARS-CoV2 infection.

\section{Materials and Methods}

\section{Study design}

We conducted a cross sectional observational descriptive analysis in a cohort of 102 patients with positive SARS-CoV-2 nucleic acid test results who were hospitalized in COVID-19 dedicated facilities of SMS Hospital, Jaipur from $19^{\text {th }}$ March to $15^{\text {th }}$ April 2020.

The people who had history of travel outside the country, respiratory symptoms or fever, resident of hotspot areas or contact with confirmed COVID-19 positive case underwent nasopharyngeal and oropharyngeal testing for SARS-CoV-2 confirmation. Tests were conducted using real-time reverse transcription polymerase chain reaction (rRT-PCR) using WHO approved kits. Patients were considered to have confirmed infection if the initial test result was positive and then confirmed with a nucleic acid sequencing.

\section{Data collection}

For each case, detailed history including travel history of self and family members and contacts was obtained. Information regarding age, sex, previous medical history, clinical manifestations, and vaccination was obtained. A detailed epidemiological history was taken for every index case and contact identification, listing and follow up was done. A close contact was defined as per guidelines issued by ministry of health and family welfare, Government of India [13]. Mobile phone locations of index cases for previous 14 days were also used to determine contacts. All contacts were then quarantined and subjected to SARS-CoV-2 testing by rRTPCR at the beginning and at the end of quarantine period or whenever they become symptomatic. All those who tested positive were isolated at SMS hospital, Jaipur in a separate designated facility.

\section{Clinical management}

All positive cases were subjected to routine hematological and biochemical blood tests, electrocardiogram (ECG) and chest radiography. RT-PCR for H1N1 was sent in all patients with bilateral infiltrates on chest x-ray. Other investigations like 2Dechocardiogram, ultrasound of abdomen, computed tomography (CT) scan were done as indicated clinically. Radiological findings, treatment measures, and prognosis were gathered after detailed examination of patients and from electronic patient record system. Apart from clinical monitoring, blood oxygen saturation $\left(\mathrm{SPO}_{2}\right)$ monitoring by a handheld pulse oxymeter was also done in each case at four-hourly intervals. The patients were put on treatment as per their clinical condition, decided by the Institutional COVID treatment protocol team. All the cases were given hydroxychloroquine (400 mg twice daily on first day and then 200 $\mathrm{mg}$ twice daily for four days) unless contraindicated. Other drugs e.g., antihistaminics and antipyretics were given on symptom basis. Patients with fever and leucocytosis and/or lung infiltrates on chest radiography were started on antibiotics like azithromycin or a cephalosporins. Antiretroviral drugs (lopinavir and ritonavir 200 $\mathrm{mg} / 50 \mathrm{mg}$; two tablets twice daily for 7-14 days) were given to selected patients with moderate disease and associated comorbidities. High flow $\mathrm{O}_{2}$ via nasal cannula or high flow mask was given in intensive care unit (ICU) to those who failed to maintain $\mathrm{SpO}_{2}<94 \%$ on room air. Corticosteroids and anticoagulants were not used in any patient unless otherwise indicated as per prevailing guidelines at that time. rRT-PCR for COVID-19 was done every third day till the test was negative, then it was repeated after $24 \mathrm{~h}$ (as per the prevailing guidelines at that time) [13]. A patient was termed cured/recovered if he became asymptomatic along with two negative rRT-PCR tests 24 hours apart. The patients were followed till any of the outcomes (cured/discharged with home isolation/died) was achieved. All data was recorded in a predesigned case record form (CRF). The patients with incomplete records or the patient who were transferred to other facilities before becoming negative were excluded from the study.

\section{Statistical analysis}

Statistical analysis was performed using the IBM SPSS ${ }^{\circledR}$ Statistics 24.0. The normality test for continuous variables was done and data was found to be normally distributed. For non-normal data Mann-Whitney test was applied between the groups. Results of continuous variables were expressed as mean $\pm \mathrm{SD}$. The independent $t$-test was applied to analyse the differences in variables between groups; $p$-values of $<0.05$ were considered significant.

\section{Results}

A total of 102 patients with confirmed SARS-CoV-2 infection were enrolled in this study. There were $77(75.49 \%)$ males and 25 $(24.51 \%)$ females. The mean age of the study population was $38.18 \pm 17.55$ years (age range: $10-85$ years). Ten patients were aged more than 65 years (Figure 1). The mean age of the male patients was $38.12 \pm 17.62$ years and that of the female patients was $38.27 \pm 17.90$ years $(\mathrm{p}>0.05)$. Eighteen $(17.65 \%)$ cases had associated comorbidities; the most prevalent were diabetes mellitus $10.8 \%$ $(11 / 102)$, hypertension $7.8 \%(8 / 102)$ and chronic obstructive pulmonary disease in four $(3.92 \%)$ cases. Chronic kidney disease, cardiovascular disease and cerebro-vascular disease were present in one patient each. Eighty-five out of 102 cases were clinically 
asymptomatic while remaining 14 presented with symptoms or developed them during hospital stay. The presenting symptoms ranged from mild upper respiratory illness to respiratory distress and multi organ failure (Table 1$)$. Ninety-nine $(88.2 \%)$ cases were managed in isolation wards while $12(11.76 \%)$ patients were shifted to ICU. Out of these 12 patients, nine $(8.82 \%)(9 / 102)$ were able to maintain $\mathrm{SPO}_{2}$ via high flow mask or by non-invasive mode of ventilation (NIV) while three (2.94\%) patients required endotracheal intubation and mechanical ventilator support; all of whom died.

Apart from non-specific minor changes in some ECGs, ST elevation with $\mathrm{T}$ wave inversion suggestive of myocardial injury (vide infra) was seen in two $(1.96 \%)$ patients. The mean QTc interval was $423.88 \pm 35.78 \mathrm{msec}$ in asymptomatic while $441.89 \pm$ $39.64 \mathrm{msec}$ in symptomatic patients and was found to be statistically insignificant $(\mathrm{p}=0.066)$. QTc interval prolongation above normal reference range ( $>440 \mathrm{~ms}$ in males and $>460 \mathrm{~ms}$ in females) was seen in $16 / 102(15.68 \%)$ patients. Hydroxychloroquine was withheld in these patients. Of these 16 patients, six had underlying comorbid conditions including two with coronary artery disease.

Chest X-ray abnormalities were seen in $15.68 \%(16 / 102)$ patients of which $8.82 \%(9 / 102)$ were asymptomatic and $5.88 \%$ $(6 / 102)$ were symptomatic. These were mainly in the form of bilateral lung field infiltrates $(13 / 102,12.74 \%)$, unilateral lung infiltrates $(2 / 102,1.96 \%)$ and left sided pleural effusion in one $(0.98 \%)$ patient. None of the patient turned out to be positive for H1N1, HIV or hepatitis B or C.

The majority of cases made uneventful clinical recovery, all but three patients $(99,97.06 \%)$ turned COVID-19 negative and discharged for isolation at home/isolation centres. The mean time for a case to recover (from the day they tested positive till two negative nucleic acid reports) was $6.08 \pm 2.02$ days. Total three patients died in this cohort, one patient presented with acute intracerebral bleed and was incidentally diagnosed as having COVID-19 and died within 24 hours of admission. Other two patients had respiratory distress at the time of admission and had to be intubated immediately. One died on the second day and the other died on the $3^{\text {rd }}$ hospital day.

Hydroxychloroquine was well tolerated by all cases without any untoward adverse effects. Lopinavir/ritonavir combination was added to the treatment of only two patients with moderate disease and associated co-morbidities, and both recovered. Major clinical and laboratory parameters of COVID-19 cases in the study have been compiled in Table 2 .

\section{Atypical presentations}

One patient had atypical presentation in the form of acute coronary syndrome (ACS), ST elevation myocardial infarction (STEMI) with elevated cardiac biomarkers and 2D echocardiography revealed regional wall motion abnormality and systolic dysfunction with LVEF of $30 \%$ at rest. Another patient, a known case of hypertension and previous thrombotic stroke died because of intracranial haemorrhage (ICH).

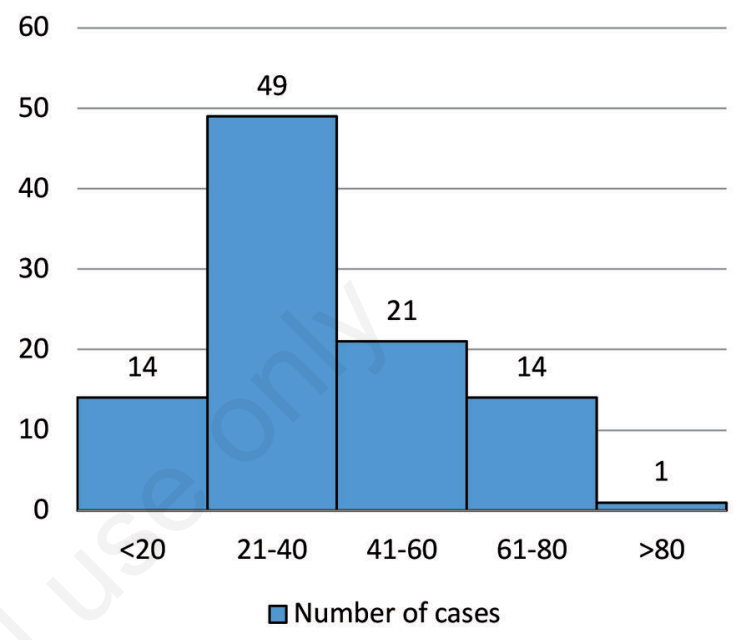

Figure 1. Age distribution of COVID-19 patients.

Table 1. Symptomatology in the 17 symptomatic COVID-19 patients.

\begin{tabular}{lc} 
Symptoms (n) & \\
Fever (11) & Chest pain (2) \\
Headache (9) & Breathlessness (10) \\
\hline Dry cough (15) & Altered sensorium (3) \\
Fatigue (9) & Haemoptysis (1) \\
\hline Running nose (7) & Nausea/vomiting (3) \\
\hline
\end{tabular}

Table 2. Major clinical and laboratory parameters of COVID-19 cases in the study.

\begin{tabular}{lcccc} 
& Total population & Asymptomatic & Symptomatic & p \\
Number of patients & 102 & $85(83.33 \%)$ & $17(16.67 \%)$ & $<0.0001$ \\
Mean age (median) (years) & $38.18 \pm 17.55(32.5)$ & $35.05 \pm 16.52$ & $53.53 \pm 14.98$ & $7.5: 1$ \\
\hline Sex ratio (m:f) & $3.08: 1$ & $2.70: 1$ & $13.12 \pm 1.58$ & 0.16 \\
Mean haemoglobin (gm/dl) & $13.73 \pm 1.99$ & $13.85 \pm 2.04$ & $8.67 \pm 3.92 \times 10^{9}$ & 0.011 \\
\hline Mean total leucocyte count (/L) & $7.38 \pm 2.32 \times 10^{9}$ & $7.12 \pm 1.782 \times 10^{9}$ & $74.47 \pm 11.89$ & 0.001 \\
Mean neutrophil count (\%) & $67.76 \pm 9.83$ & $66.42 \pm 8.85$ & $20.58 \pm 10.87$ & 0.001 \\
Mean lymphocyte count (\%) & $27.26 \pm 9.49$ & $28.60 \pm 8.65$ & $6.17 \pm 6.11$ & $<0.0001$ \\
Mean N/L ratio & $3.26 \pm 3.02$ & $2.67 \pm 1.32$ & $182.48 \pm 59.75 \times 10^{9}$ & 0.119 \\
\hline Mean platelet count (/L) & $208.71 \pm 76.58 \times 10^{9}$ & $214.28 \pm 79.08 \times 10^{9}$ & $146.29 \pm 88.90$ & 0.043 \\
Mean P/L ratio & $120.61 \pm 57.38$ & $115.47 \pm 47.88$ & $106.53 \pm 49.27$ & 0.055 \\
\hline Fasting blood sugar (FBS) (mg/dl) & $95.43 \pm 26.24$ & $93.21 \pm 18.32$ & $583.18 \pm 260.45$ & $<0.0001$ \\
Lactic acid dehydrogenase (U/L) & $439.14 \pm 166.97$ & $410.33 \pm 124.75$ & & \\
\hline
\end{tabular}




\section{Discussion}

SARS-CoV-2 has rapidly spread across many countries, although most of the COVID-19 patients have mild to moderate course, up to 5-10\% can have severe life-threatening disease course [14]. Isolating sources of infection remains an important measure to break the chain of transmission and control this pandemic. Infected individuals can be tracked according to their symptomatology, however, those who are asymptomatic, especially without an epidemiological history, are difficult to track. These asymptomatic cases may be capable of transmitting SARS-CoV-2, thus demanding isolation of such cases.

Our study is unique in the fact that all cases admitted in the hospital were traced on the basis of history of travel, contact with a confirmed COVID-19 case, resident of hotspot areas or presence of symptoms, thus providing an accurate estimate of the proportion of asymptomatic cases. In the present study, 83.33\% (85/102) cases were asymptomatic. These estimates appear more plausible owing to extensive contact tracing and sampling done in the identified "hotspot" areas. Most reliable data for the proportion of the asymptomatic cases comes from 634 positive cases on board the Diamond Princess Cruise ship where approximately $51.73 \%$ cases were asymptomatic [15]. Dry cough and fever were most common presenting symptoms in those who were symptomatic, however, loss of taste and smell sensation was not encountered in any patient. The clinical spectrum of our study was as follows: mild cases $(90 / 102$, $88.23 \%)$, moderate cases $(9 / 102,7.5 \%)$ and severe $3(2.94 \%)$ cases. Similarly, Wu et al. in their study of 72314 cases of which 44672 were confirmed cases found mild disease in $81 \%$, moderate in $14 \%$ and severe disease in $5 \%$ of their patients [16].

In the present study, $18(17.65 \%)$ patients presented with comorbidities with hypertension being most prevalent. Further, among the twelve patients with severe disease, one or more comorbid condition was present in five of them (including the three who died). Similarly, Yang et al. in their meta-analysis of 1576 patients reported hypertension $(21.1 \%)$ diabetes $(9.7 \%)$ and cardiovascular disease $(8.4 \%)$ as most prevalent comorbid conditions followed by respiratory system disease in $1.5 \%$ cases [17].

In our study radiological findings in chest x-ray were seen in 15.68 $\%(16 / 102)$ patients of whom $8.82 \%(9 / 102)$ were asymptomatic and $5.88 \%(6 / 102)$ were symptomatic, most common pattern being bilateral lung field infiltrates. Shi et al. observed that predominant radiological pattern of abnormality was bilateral lung involvement in COVID-19 cases and asymptomatic cases also had radiological findings [18]. Li et al. reported that older age, leucocytosis, and high LDH level were associated with in-hospital deaths [19]. In our study, three (2.94\%) patients died, these patients were above 65 years of age with comorbidities and biochemical profile revealed lymphopenia, higher $\mathrm{N} / \mathrm{L}$ ratio and higher $\mathrm{LDH}$ levels.

Hydroxychloroquine was administered to all patients while azithromycin was given only to symptomatic patients. It was observed that $99(97.05 \%)$ patients recovered from illness with mean time of $6.08 \pm 2.02$ days. Gautret et al. provided evidence of a beneficial effect of co-administration of hydroxychloroquine with azithromycin in the treatment of COVID-19 and its potential effectiveness in the early reduction of contagiousness and mean duration of hospital stay was reduced to 5 days [20]. Antiretroviral drugs were given to only two selected patients with moderate disease and associated comorbidities with favourable results. In a randomized controlled trial on severely ill Chinese COVID-19 patients, use of lopinavir-ritonavir failed to demonstrate any beneficial effect on clinical recovery or virologic clearance [21]. It is postulated that delayed institution of anti-viral therapy in the course of the disease may not be beneficial and the role of lopinavirritonavir needs to be further explored [22].

In eight $(7.84 \%)$ cases in our study, the subsequent rRT-PCR came positive when the prior test has been negative. This phenomenon may be attributable to false-negative results of assays or by factors involving the specimen collection, reagents, and operations and possible intermittent viral shedding, hence two consecutive negative samples more than $24 \mathrm{~h}$ apart should only be taken as discharge/end of isolation criterion [23]. There were no readmissions among the discharged patients till the date of publishing this study.

The infectiousness and effect of the asymptomatic epidemiological characteristics and disease clinical characteristics of dynamic change are not clear. It appears that close monitoring of confirmed COVID-19 cases and administration of hydroxychloroquine early in the course of the disease led to early recovery and better outcomes in these patients.

\section{Limitation of study}

The sample size of study population was small.

\section{Conclusions}

With majority of COVID-19 cases in India being asymptomatic, there is little research on asymptomatic epidemiology, biochemical and radiological profile especially in Indian COVID-19 cases. The patients in the study did not require prolonged hospital stay and the fatal complications were seen early in the clinical course. Elevated NLR, lymphopenia, age and presence of comorbidities were associated with increased severity and poor outcome.

\section{References}

1. WHO. Pneumonia of unknown cause China. Emergencies, preparedness, response, disease outbreak news. World Health Organization. 2020. Accessed: 8 May 2020. Available from: https://www.who.int/csr/don/05-january-2020-pneumonia-ofunkown-cause-china/en/

2. Centers for Disease Control and Prevention. 2019 Novel Coronavirus, Wuhan, China. CDC. Accessed: 27 January 2020. Available from: https://www.cdc.gov/coronavirus/2019-ncov/ about $/ \% 20$ index.html

3. WHO. Director-General's remarks at the media briefing on 2019nCoV on 11 February 2020. World Health Organization. 2020. Accessed: 4 May 2020. Available from: https://www.who. $\mathrm{int} / \mathrm{dg} / \mathrm{speeches} /$ detail/who-director-general-s-remarks-at-the media-briefing-on-2019-ncov-on-11-february-2020

4. Coronaviridae Study Group of the International Committee on Taxonomy of Viruses. The species severe acute respiratory syndrome-related coronavirus: classifying 2019-nCoV and naming it SARS-CoV-2. Nat Microbiol 2020;5:536-44.

5. WHO. Coronavirus pandemic. World Health Organization. 2020. Accessed: 25 July 2020. Available from: https:/www.who.int/ emergencies/diseases/novel-coronavirus-2019

6. Government of India [Internet]. \#IndiaFightsCorona COVID-19. 
2020. Accessed: 25 July 2020. Available from: https://www. mygov.in/COVID-19

7. Huang L, Zhang $X$, Zhang $X$, et al. Rapid asymptomatic transmission of COVID-19 during the incubation period demonstrating strong infectivity in a cluster of youngsters aged 16-23 years outside Wuhan and characteristics of young patients with COVID-19: A prospective contact-tracing study. J Infect 2020;80:e1-13.

8. Chen N, Zhou M, Dong X, et al. Epidemiological and clinical characteristics of 99 cases of 2019 novel coronavirus pneumonia in Wuhan, China: a descriptive study. Lancet 2020;395:507-13.

9. Huang C,Wang Y, Li X, et al. Clinical features of patients infected with 2019 novel coronavirus in Wuhan, China. Lancet 2020;395: 497-506.

10. Kolifarhood G, Aghaali M, Mozafar Saadati H, et al. Epidemiological and clinical aspects of covid-19; a narrative review. Arch Acad Emerg Med 2020;8:e41.

11. Yao X, Ye F, Zhang M, et al. In vitro antiviral activity and projection of optimized dosing design of hydroxychloroquine for the treatment of severe acute respiratory syndrome coronavirus 2 (SARS-CoV-2). Clin Infect Dis 2020;71:732-9.

12. Chan JF, Yuan S, Kok KH, et al. A familial cluster of pneumonia associated with the 2019 novel coronavirus indicating person-toperson transmission: a study of a family cluster. Lancet 2020; 395:514-23.

13. Ministry of Health and Family Welfare, Government of India. Guidelines on the clinical management of COVID-19. Accessed: 8 May 2020. Available from: https://www.mohfw.gov.in/ pdf/GuidelinesonClinicalManagementofCOVID1912020.pdf

14. Şimşek yavuz S, Ünal S. Antiviral treatment of COVID-19. Turk J Med Sci 2020;50:611-9.

15. Mizumoto K, Kagaya K, Zarebski A, Chowell G. Estimating the asymptomatic proportion of coronavirus disease 2019 (COVID19) cases on board the Diamond Princess Cruise ship, Yokohama, Japan, 2020. Euro Surveill 2020;25:2000180.

16. Wu Z, McGoogan JM. Characteristics of and important lessons from the coronavirus disease 2019 (COVID-19) outbreak in China: Summary of a report of 72314 cases from the Chinese center for disease control and prevention. JAMA 2020; 323:1239-42.

17. Yang J, Zheng Y, Gou X, et al. Prevalence of comorbidities and its effects in coronavirus disease 2019 patients: A systematic review and meta-analysis. Int J Infect Dis. 2020;94:91-5.

18. Shi H, Han X, Jiang $N$ et al. Radiological findings from 81 patients with COVID-19 pneumonia in Wuhan, China: a descriptive study. Lancet Infect Dis 2020;20:425-34.

19. Li X, Xu S, Yu M, et al. Risk factors for severity and mortality in adult COVID-19 inpatients in Wuhan. J Allergy Clin Immunol 2020;146:110-8.

20. Gautret P, Lagier JC, Parola P, et al. Clinical and microbiological effect of a combination of hydroxychloroquine and azithromycin in 80 COVID-19 patients with at least a six-day follow up: A pilot observational study. Travel Med Infect Dis 2020;34:101663.

21. Cao B, Wang Y, Wen D, et al. A trial of lopinavir-ritonavir in adults hospitalized with severe COVID-19. N Engl J Med 2020;382:1787-99.

22. Baden LR, Rubin EJ. Covid-19 - The search for effective therapy. N Engl J Med 2020;382:1851-2.

23. European Centre for Disease Prevention and Control. Guidance for discharge and ending isolation in the context of widespread community transmission of COVID-19, 8 April 2020. Stockholm: ECDC; 2020. Available from: https://www.ecdc.europa.eu/en/ publications-data/covid-19-guidance-discharge-and-ending isolation 